\title{
Modelling and control of a jet mill plant
}

\author{
H.J.C. Gommeren *, D.A. Heitzmann, J.A.C. Moolenaar, B. Scarlett \\ Laboratory for Process Equipment, Leeghwaterstraat 44, 2628 CA Delft, Netherlands
}

Accepted 20 September 1999

\begin{abstract}
Although jet milling is a very energy consuming grinding process it is increasingly used in industry because very fine grinding product with a narrow size distribution is attained without contamination as the milling occurs by inter particle collisions. At Delft University of Technology a project has been started to achieve a considerable energy reduction in jet milling processes. The grinding plant consists of a spiral jet mill in closed loop with an external classifier. Main feature of the system will be an operation control based on in-line particle size measurements using laser diffraction. In industrial practice the operating conditions are often determined by trial and error. To avoid off-spec material the mill is often tuned to lower risk. This results in a relatively large amount of overground material. With respect to the control strategy, the first step was real time particle size monitoring to explore the operating ranges of the jet mill. The controlability is studied in relation to several process inputs and process configurations. A dynamic model of the closed loop grinding plant is developed. Particle transport and size reduction inside the mill show a stochastic behaviour and are described by size and state dependent probability functions. Separate experiments are carried out to derive equations for the breakage kinetics of different materials. Numerical flow simulations are carried out to provide statistic data about the frequency and intensity of collisions between particles in relation to state conditions in the mill. A glass bottom plate will be placed on the mill to observe flow patterns. The influence of several process input variables on the dynamics of the grinding plant and the final product are simulated. Pilot plant experiments are carried out to verify and optimize the dynamic model by direct measurement of the PSD under actual system conditions. The ultimate objective of the dynamic model will be the implementation in a control system. The required setpoints of the mill/classifier system are predicted to obtain the desired product quality at minimum energy use. Furthermore the model can be used for scale up and plant design. (C) 2000 Elsevier Science S.A. All rights reserved.
\end{abstract}

Keywords: Jetmilling; Modelling, simulation; Control; In-line particle size measurement; Computational fluid dynamics

\section{Introduction}

Since the main disadvantage of jet milling is the high amount of energy required for the grinding operation, the aim of this project is a 50\% reduction of the energy consumption. The basic idea for meeting this objective is operation of the jet mill in closed circuit with an air classifier (see Fig. 1). For adequate process control it is necessary to have a dynamic model of the mill and classifier system to be applied in this control system.

The development of an overall plant model requires insight in the breakage kinetics of the material and how the particles are submitted to loading forces in the milling chamber. Fig. 2 shows at which levels the modelling is

\footnotetext{
* Corresponding author. E-mail: gomm@dutw110.tudelft.nl
}

being developed. Level 1 is the microscopic level, where the breakage of a single particle is considered as a function of its mechanical properties, size and supplied energy. Single particle breakage tests are done to access some of the parameters in the comminution functions. Level 2 concerns flow patterns in the milling chamber providing information about the collision intensity and transfer of particles.

The first two models are used to get insight in the phenomena that play a role in the macroscopic process (level 3). This model is based on a physical description of the processes completed with experimental data. For different grinding materials, mill geometries and process inputs, the behaviour of the plant is to be predicted allowing plant design and better control. The optimal combination of setpoints in the grinding plant is estimated by this 


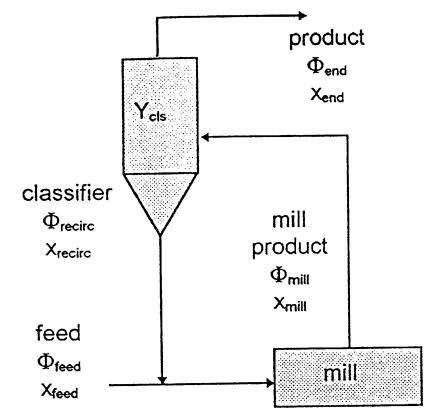

Fig. 1. Closed circuit grinding system.

model in such a way that the desired product quality will be achieved with minimum energy use.

The three models are described in this paper.

\section{Description of the pilot plant}

\subsection{Apparatus}

\subsubsection{Jet mill}

Several types of jet mills are used in industry. The one investigated in this project is a 10-in. spiral jet mill. The maximum capacity of this mill is $25 \mathrm{~kg} / \mathrm{h}$ at 7 bar. In Fig. 3 a schematic picture is shown of such a mill. Solid feed (plastic granulate) is blown into the mill chamber by an air-pusher. High pressure air enters the mill through a number of nozzles placed around the cylindrical chamber. The particles to be ground are accelerated up to high velocities $(200 \mathrm{~m} / \mathrm{s})$. Collisions between the particles in the highly turbulent jets result in comminution. A rotating stream is created by the direction of the nozzles. Particles in this flow field are submitted to centrifugal and drag forces. Larger particles are transferred to the outer zone, while small particles are dragged to the central outlet. This phenomena is called internal classification of the mill.

\subsubsection{Air classifier}

At higher throughputs large fluctuations in the product quality appear. Optimization for grinding and internal classification performance of a spiral jet mill cannot be ob-

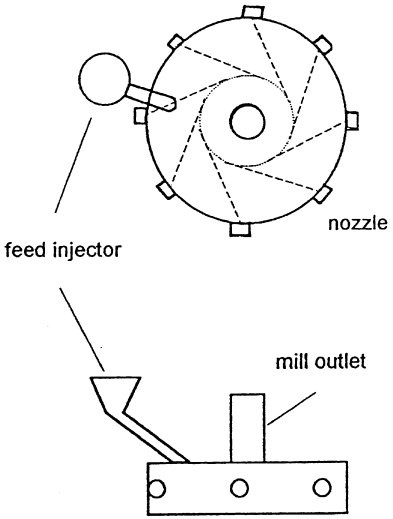

Fig. 3. A spiral jet mill.

tained simultaneously. Therefore an external classifier has been installed on the mill outlet. The dispersed powder flow leaving the mill is split into coarse fraction which is recirculated and fines which are collected in a bag filter as end product This is called a closed circuit grinding system (see Fig. 1).

\subsection{Control system}

In Fig. 4 the technical-functional lay out of the control system is shown. The tasks of the distributed control system are described below.

\subsubsection{Monitoring}

The ETP software package, installed on PC1, serves as a monitoring and control platform. Process information is presented by trending screens, bar diagrams and reports with numerical values. A graphical display (animation screens) is designed which comprises a main screen displaying all important process parameters and sub screens which contain information on a specific grinding plant.

\subsubsection{Plant automation}

Local PID controllers and sensors in the grinding plant are connected to the process computer (PC1) by a RS485 interface. After discussions with plant operators and pro-

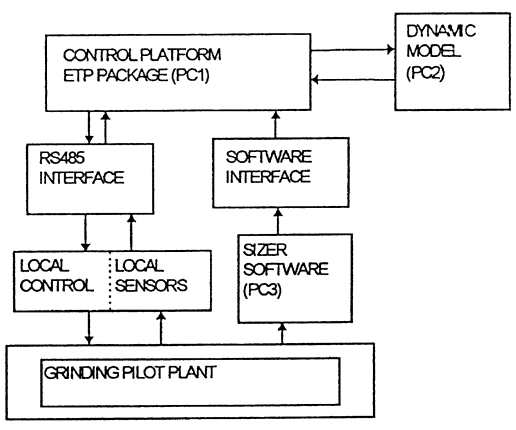

Fig. 4. Architecture of measurement and control system.

Fig. 2. Levels of modelling. 
duction staff procedures for automatic start-up and shutdown are set up and implemented in the control software.

\subsubsection{Control}

Model calculations are carried out on PC2 estimating the values of the process variables. Ultimately the model has to predict the optimum setpoints of the process which are downloaded to the PID controllers in the field. Experimental data will be transmitted to the computer for on-line optimization/adaptation of the dynamic model.

\subsubsection{Sensors}

An in-line cell for real-time measurements of the particle size distribution of the powder leaving the mill was built using laser diffraction (see Fig. 5). The mill product dispersed in air flows through the optical cell. The scattering of an incident laser beam by a collective of particles in the sampling volume is measured on 31 wedge detectors.

The theoretical expected light diffraction pattern of a known particle distribution is described by the Fraunhofer theory. Discretisation of the diffraction model results in the following linear output model:

$L=G n$

where $G$ the sensor model matrix, $L$ the scattered light energy vector measured on 31 detector wedges and $n$ a discrete population density.

Interpretable quantities, for example the $X_{90}$, the median particle size and width of the size distribution can be calculated by deconvolution from the diffraction pattern. Deconvolution calculations can introduce inaccuracies in the estimated size distribution. An alternative is to use raw detector data for control purposes. These options are discussed in Section 2.3 'control strategy'.

\subsubsection{Actuators}

As process actuators, respectively the solid feedrate $\left(\Phi_{\text {feed }}\right)$ and the air pressure on the nozzles $\left(P_{\text {nzl }}\right)$ are considered. The supplied high pressure air is a measure for the energy consumption, as the feedrate determines the throughput of the plant. In the case of a closed loop grinding circuit the classifier efficiency $\left(Y_{\mathrm{cls}}\right)$ can be adjusted, providing an extra handle to control the process.

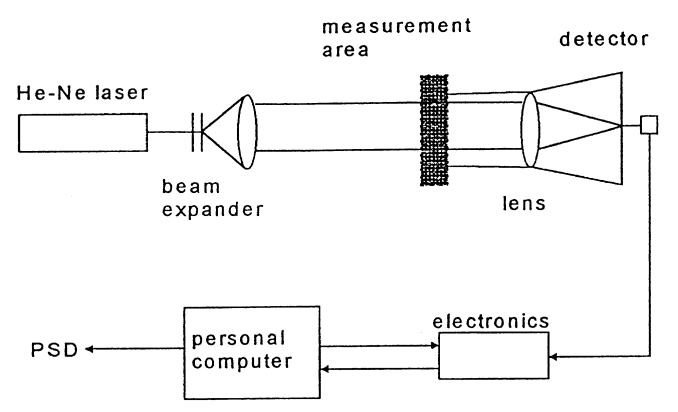

Fig. 5. Laser diffraction aperture.

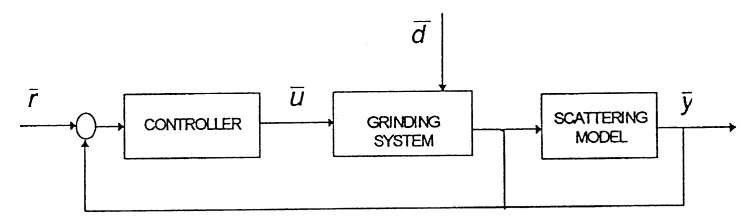

Fig. 6. Scheme of feedback system (where $r$, reference; $u$, inputs; $d$ disturbances; and $y$, outputs of the grinding system).

\subsection{Control strategies}

\subsubsection{Control objectives}

The product specification is defined as an aspect of the particle size distribution. If the ground product is finer than the maximum allowed size the product is usually acceptable. The objectives are:

(a) Maintaining the desired product quality (e.g., $X_{90}$ or $\left.X_{50}\right)$.

(b) Process optimization in relation to product quality and energy consumption

The setpoints of the controllers in the grinding plant are being adjusted in such a way that these objectives are met, using in-line particle size measurements.

\subsubsection{Choice of controller inputs}

(a) The laser diffraction instrument measures light intensities on 31 detectors which are sent to a PC to be converted into a particle size distribution. Generally sensor models are ill conditioned and therefore the calculation of the PSD from light intensities may give inaccurate results. It will be investigated whether the processed PSD or raw detector signals should be applied as a controller input(see Fig. 6).

(b) Furthermore signals from other sensors (e.g., pressure level in the mill chamber) are studied to serve for control.

(c) Observer: An important application of the dynamic process model is to serve as a state estimator. Dynamic process data stored implicitly in a dynamic model is combined with on-line process information to reconstruct quantities that cannot be measured real-time (e.g., hold-up) or only with great difficulty.

\subsubsection{Feedback on the median particle size $\left(X_{50}\right)$}

A control has been realized using the $X_{50}$ as a feedback signal to the solid feedrate (see Fig. 6). Low frequency

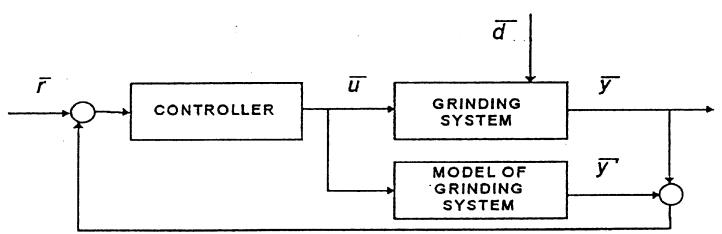

Fig. 7. Scheme of model predictive control included with an observer(where $u$, inputs; $d$, disturbances; $x^{\prime}$, estimated internal variables; $y^{\prime}$, estimated output variables; and $y$, outputs of the grinding system). 
disturbances $(d)$ in the air supply or changes in grindability are compensated for. Applying this SISO control alone allows the achievement of the first objective.

\subsubsection{Model predictive control}

Pilot plant experiments showed that several combinations of manipulated variables exist (feedrate, pressure, classifier cutsize) resulting in the same product quality. To satisfy the second objective, system knowledge implemented in the dynamic model is required to estimate the optimum combination of setpoints (see Fig. 7).

\section{Modelling}

When the model will solely be used for stabilizing feedback controller design, accurate estimates of the kinetics are not necessary. For process design or observer design often more detailed models with a larger accuracy are needed [2]. The sources of information to develop the model are:

- Understanding of the main mechanisms of the process by rigorous modelling

- Experience based information obtained by interview-

ing operators

- Carrying out pilot plant experiments.

Fig. 2 shows that the modelling is divided into three sub-models. Section 3.1 deals with the main model (level 1) predicting the dynamic interaction of the jet mill/classifier system. Section 3.2 explains about the flow simulations that calculates the motions and collisions of individual particles in the mill chamber. The breakage of a single particle is modelled in Section 3.3.

\subsection{Dynamic model of jet mill / air classifier system}

In a jet mill two major phenomena take place; comminution and transfer of particles, with their dependence one upon the other. The amount of particles present inside the mill chamber (hold up) is an important state variable as the frequency and intensity of collisions determines the grinding efficiency of the mill. The internal classification depends on the stability and force of the vortex flow, which is related to the hold-up of the mill as well.

The performance of the jet mill plant considered here is described by a population balance model calculating the evolution in time of the PSD of the material present in different zones in the plant (Fig. 8). For a detailed description of the development of the dynamic model one is referred to the preprints of the 8th Eur. symp. on comminution [3]. Mass balances per size class $(i)$ over the zones contain statistical functions for particle breakage and transfer. After each time step the balances are calculated for each zone, providing information about the hold-up,

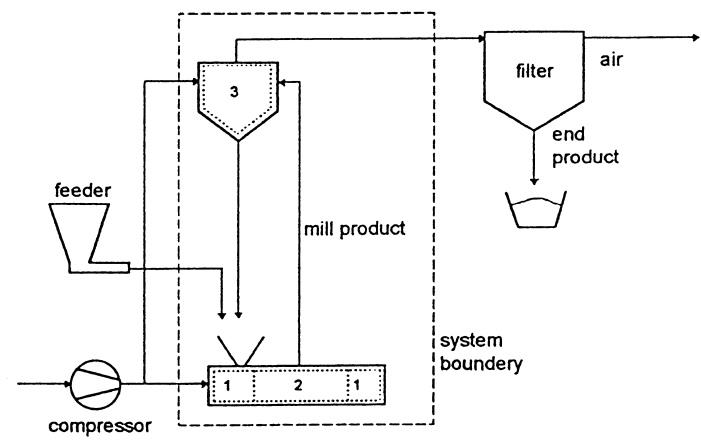

Fig. 8. System boundary of dynamic model. The system is divided into three subsystems.

flowrate and the PSD. The population balance over zone 1, the outer zone of the mill chamber, is:

$$
\begin{aligned}
m_{1, i}(t+\Delta t)= & m_{1, l}(t)+m_{2, i}(t) P_{21, i} \Delta t \\
& +\sum_{j=1}^{i} b_{i, j} S_{j} m_{1, j}(t) \Delta t-m_{1, i}(t) P_{12, i} \Delta(t) \\
& -S_{i} m_{1, i}(t) \Delta t
\end{aligned}
$$

where $m_{1, i}$ mass of size fraction $i$ in zone $1, m_{2, i}$ mass of size fraction $i$ in zone 2 .

\subsubsection{Particle transfer}

The particle transfer in the mill chamber is described by S-shaped functions $\left(P_{12, i}\right)$ which determine the probability that a particle with size $x$ is removed from the mill. These empirical equations for particle transfer are parametrized by a cutsize and a sharpness. These parameters are related to the hold-up in the zones and the supplied air. The asterisk $(*)$ indicates the nominal value, in a situation where there is no particle-particle interaction (hold-up $M_{2}$ low). Thus a higher hold-up leads to a larger $X_{50}$ and a wide spread.

$$
\begin{aligned}
& P_{23 i}=K_{23}\left(1-\frac{1}{2}\left(1+\operatorname{erf}\left(\frac{\ln x_{i}-\ln x_{50 \_23}(t)}{\sqrt{2} \ln \sigma_{23}(t)}\right)\right)\right) \\
& x_{50 \_23}(t)=x_{50 \_23}^{*}\left(1+K_{x 23} M_{2}^{2}(t)\right) / P_{\mathrm{nzl}}^{0.5} \\
& \text { and } \sigma_{23}(t)=\sigma_{23}^{*}\left(1+K_{s 23} M_{2}^{2}(t)\right)
\end{aligned}
$$

where $K_{23}, K_{x 23}, K_{s 23}$ are constants.

\subsubsection{Size reduction}

The probability for a particle to break within a time step is expressed by the selection for breakage function $S_{i}$ (see Fig. 9). The classical equation [6] is extended with an empirical term to take into account the influence of the loading conditions. Each type of material and size has its own optimum hold up for obtaining the largest selectivity for breakage. Hold-ups higher or lower will result in insufficient collisions and thus result in less efficient grind- 


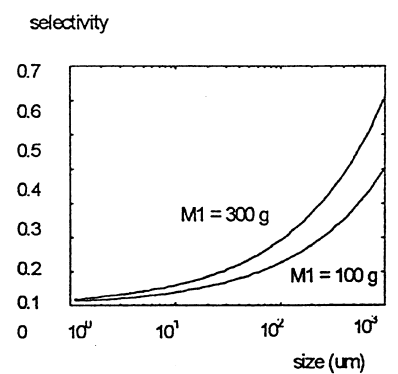

Fig. 9. Selectivity for breakage for different hold ups (M1) in zone 1.

ing. The nozzle pressure is a measure for the supplied kinetic energy to the material to be ground.

$S_{i}=\frac{K_{s 1} M_{1}}{K_{s 2}+M_{1}^{1.5}} P_{\mathrm{nzl}}^{2}\left(\frac{x_{i}}{x_{\max }}\right)^{0.5}$

where $K_{s 1}, K_{s 2}$, constants [-]; $M_{1}$ hold up zone $1[\mathrm{~g}] ; x_{1}$ particle size $[\mu \mathrm{m}], P_{\mathrm{nzl}}$ nozzle pressure [bar].

$B_{i j}$ is the breakage function that describes how fragments of a broken particle $(j)$ are divided over the smaller size classes $(i)$. Fig. 10 shows three-dimensional plots of the breakage function. The shape of this function is derived from off-line particle breakage tests (see par. 4.2). Different modes can be distinguished depending on the breakage phenomena. Attrition is the main mechanism in jet milling as the applied forces during the collisions are mostly restricted to a partial volume at the surface. The result is that after breakage the original particle remains large and rounded. Attrition results in an extra peak in the fines region (marked with a, Fig. 10), as normal breakage shows a wider curve $(b)$.

$$
\begin{aligned}
B_{i j}= & \frac{\Phi}{2}\left[1+\operatorname{erf}\left(\frac{\ln \left(\mathrm{rf}_{1} x_{i} / x_{j}\right)}{\ln \sigma_{1} \sqrt{2}}\right)\right] \\
& +\frac{1-\Phi}{2}\left[1+\operatorname{erf}\left(\frac{\ln \left(\mathrm{rf}_{2} x_{i} / x_{j}\right)}{\ln \sigma_{2} \sqrt{2}}\right)\right]
\end{aligned}
$$

In which $\mathrm{rf}_{1}, \mathrm{rf}_{2}$ breakage modes $[-], \sigma_{1}, \sigma_{2}$ variance around modes $[-], \Phi$ constant $[-]$.

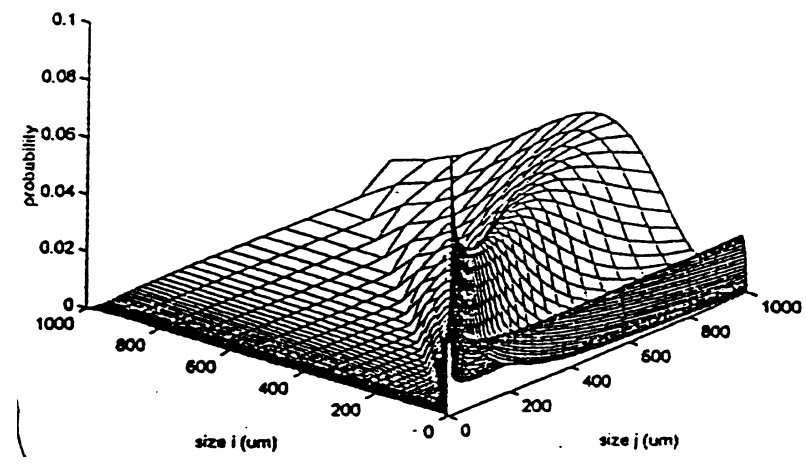

Fig. 10. Breakage function $B_{i j}$.

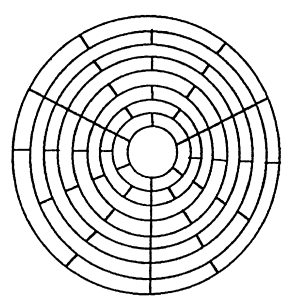

Fig. 11. Size grid of simulation.

\subsubsection{External classifier}

The performance of the external classifier is described by the Plitt equation of which the parameters are determined by pilot plant experiments.

\subsection{Flow patterns in mill chamber}

In this paragraph the modelling on level 2 (Fig. 2) is described.

\subsubsection{Simulation method}

The simulation method (DSMC) for solid/liquid deals with both phases separately. Flow of the solid phase is obtained by calculating individual particle motions, while the gas flow is obtained by solving the equations of viscid fluid flow. This method is used to take account of particle-particle collisions.

\subsubsection{Flow field}

The flow field in the mill chamber is divided into small cells in which the change of flow properties is small. Since the simulation method requires that all cells are of approximately equal size, the grid is chosen as shown in Fig. 11. The particles are allowed to collide by a Monte Carlo procedure. (A Monte Carlo method can be classified as a probabilistic method). The motions of all simulated particles within time step $\Delta t$ are calculated by the equations of motion without regard for inter particle collisions; if the particle crosses a solid wall, the velocity is replaced by the post-rebound velocity. Inter-particle collisions during the time-interval $\Delta t$ are examined by means of a Monte Carlo procedure. The post-collision velocities of the collision pair are calculated by the impulsive equations.

The flow problem is reduced to a two-dimensional problem, using a circular configuration. During the process particles are added through the injector at a prescribed feed rate. Particles can exit the calculation region through the central outlet pipe. The particle concentration is so low that the gas velocity distribution is not affected by the solid particles. Thus coupling between solid and fluid phases only exists in one direction. The fluid velocity consists of a radial and a tangential component, which both are a function of the radial coordinate only. For the fluid flow the following equations are used:

$u_{\mathrm{f}, r}=\frac{C_{u, r}}{r}$ and $u_{\mathrm{f}, \theta}=\frac{C_{u, \theta}}{r^{0.6}}$ 


\subsubsection{Forces on particles}

The equation of particle motion follows from Newton's second law and is given by:

$m_{\mathrm{p}}=\frac{\mathrm{d} u_{\mathrm{p}}}{\mathrm{d} t}=\sum F_{\mathrm{f}}=F_{\mathrm{C}}+F_{\mathrm{D}}+F_{\mathrm{M}}+F_{\mathrm{L}}$

The centrifugal force $F_{\mathrm{C}}$ is calculated from the expression:

$F_{\mathrm{C}}=m_{\mathrm{p}} \frac{u_{r, \theta}^{2}}{|r|^{2}} r$

The drag force $F_{\mathrm{D}}$ acting upon the particle is given by:

$F_{\mathrm{D}}=0.5 C_{\mathrm{D}} A_{\mathrm{p}} \rho_{\mathrm{p}}\left|u_{\mathrm{R}}\right| u_{\mathrm{R}}$

For the other terms one is referred to the report "computer simulations of a jet mill' [4].

\subsubsection{Particle collisions}

In this simulation it is assumed that binary collisions are dominating. Examination of inter-particle collision in every time step is based on the modified Nanbu procedure [7]. The occurrence of inter-particle collision and the collision partner are decided by using random numbers which are obtained from a uniform distribution in the range from zero to unity. The probability of particle $i$ to collide with another particle during a time step $\Delta t$ is given by:

$P_{i}=\sum_{i=1}^{N} P_{i j}$

where $N$ is the number of simulated particles in the cell and $P_{i j}$ is the probability of collision between particle $i$ and $j$ during time step $\Delta t . P_{i j}$ is given by:

$P_{i j}=\frac{n}{N} \pi d_{\mathrm{P}}^{2} u_{\mathrm{R}} \Delta t$

A candidate collision partner is selected using the Nanbu method, following equation:

$k=\left[\left[R_{\mathrm{ND}} N\right]\right]+1$

The condition that must be satisfied when a collision with particle $j$ actually takes place in the previous time step is:

$R_{\mathrm{ND}}>\frac{k}{N}-P_{i k}$

When a particle collides with another particle or with the wall, the post-collision velocities $(*)$ are calculated on the basis of the equations of impulsive motion:

$u^{*}=u+\frac{J}{m_{\mathrm{P}}}$ and $\omega^{*}=\omega+0.5 \frac{d_{\mathrm{P}}}{I} n J$

The coefficient of restitution is constant and the tangential force during the motion is based on Coulomb's law of friction. In that case the following equations for the impulsive force, $J$, is valid:

$J=J_{n} n+J_{t} t$

$J_{n}=\left(1+e_{\mathrm{p}}\right) M n u_{\mathrm{R}}$ and $J_{t}=\min \left[-f_{\mathrm{p}} J_{n}, \frac{2}{7} M\left|u_{\mathrm{s}}\right|\right]$ $t$ is the tangential unit vector. $u_{\mathrm{s}}$ is the slip velocity of the collision partner with respect to the particle under consideration, $f_{\mathrm{p}}$ is the coefficient of friction and $M$ is given by:

$M=\frac{m_{\mathrm{p}, 1} m_{\mathrm{p}, 2}}{m_{\mathrm{p}, 1}+m_{\mathrm{p}, 2}}$

In this equation $m_{\mathrm{p}, 1}$ and $m_{\mathrm{p}, 2}$ are the masses of the two particles involved in the inter-particle collision. In the case of collision with a wall $M=m_{\mathrm{p}}$.

\subsubsection{Particle breakage}

Not every particle in the mill will break upon colliding with another particle or with the wall. The selection function and breakage functions used in this model are the same as the functions used in the dynamic model (see Section 4.1). The selection function is extended with a term that takes in account the kinetic energy of the collision.

\subsubsection{Simulation results}

The first version of this program has now been completed. The next steps will be to carry out more extensive test runs and to assess the influence of several parameters on the outcome of the simulations, such as cell size, number of particles and the time step. The flow simulations will be verified with laser doppler velocity measurements.

\subsection{Simulation of single particle breakage}

This paragraph deals with the modelling at level 3 of Fig. 2. The breakage of a particle is considered with respect to the collision energy as a function of its size and physical properties. The models simulate under which conditions breakage occurs and how a particle fragments.

\subsubsection{Simulation of single particle breakage [5]}

With the use of a simulation program for brittle-elastic fracture [8] the single particle breakage of our test material was simulated. A breakable particle is created by 'glueing' together unbreakable and non-deformable solid elements which do not deform on contact with a wall or other elements. Instead, the contacting surfaces overlap slightly and produce a restoring force that is proportional to the overlap (see Fig. 12).

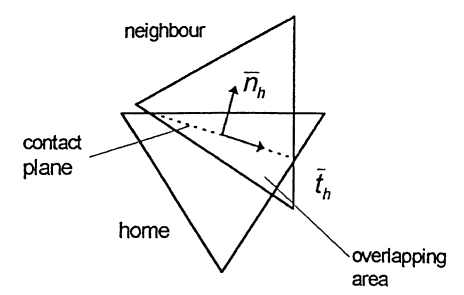

Fig. 12. Simulation of single particle breakage. 


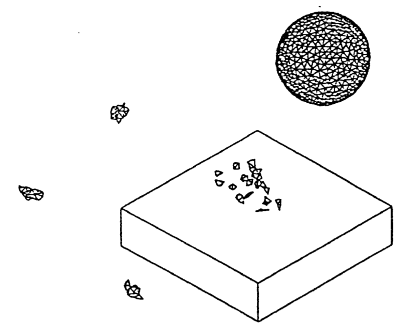

Fig. 13. Simulation of breakage of a single particle by impact on a plate.

A glued joint that connects the edges of two adjacent polygons is modelled as a set of elastic fibers that connect initially coincident points on the two edges. Each such "fiber" has stiffness $K_{\mathrm{n}}$. The normal force is $K_{\mathrm{n}}$ times the length of the projection of the fiber onto the direction normal to the element surfaces. Similarly, the tangential force is the tangential projection of the fiber multiplied by the tangential stiffness $K_{\mathrm{t}}$. Thus the composite body is deformable, even though its constituent particles are not, due to the compliance of the joint.

The Young's modulus and Poissons ratio of the composite depend on $K_{\mathrm{n}}$ and $K_{\mathrm{t}}$. The composite material may fracture along the joints between the elements. A glued joint can withstand normal tensile stress up to the limit $s_{\text {tens }}$ If tensile stress on any portion of the joint exceeds $s_{\text {tens }}$, the 'glue' along that portion breaks; in other words, a crack forms along that portion of the joint which is at least $s_{\text {tens }}^{2} / 2 K_{n}$ per unit length. Energy is stored in the normal displacement of the joint.

The force tangential to the contact is elastic and proportional to the relative displacement of the contacting particles with the coefficient of $K_{\mathrm{t}} L_{\mathrm{p}}$, where $L_{\mathrm{p}}$ is the length of the particle side, up to a frictional limit corresponding to a friction coefficient, $\mu$.

A particle, of characteristic size, $L_{\mathrm{o}}$, strikes an unbreakable rigid target with velocity $V_{0}$. Before collision the particle is completely unstressed. The strength of the material is related to the violence of the impact. This is defined as a ratio of the kinetic energy of the impact to the minimum energy lost in creating a crack that spans the

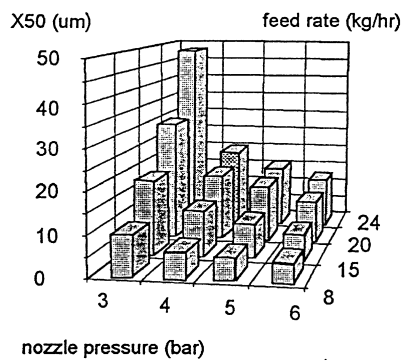

Fig. 14. $X_{50}$ depending on feedrate and nozzle pressure (experimental results).

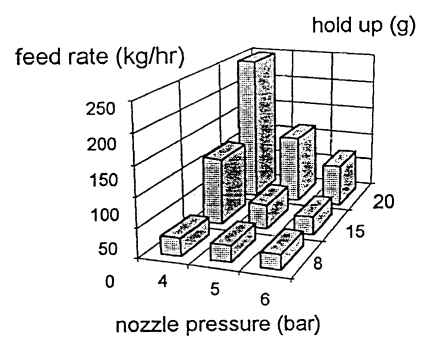

Fig. 15. Hold up depending on feedrate and nozzle pressure (experimental results).

particle. The larger $E_{\mathrm{kln}} / E_{\mathrm{cr}}$, the greater the damage the particle should experience. In formula:

$E_{\text {kin }}=\left(\rho \pi L_{\mathrm{o}}^{3} \nu_{\mathrm{o}}^{2}\right) / 8$ and $E_{\mathrm{cr}}=\left(\sigma_{\text {tens }}^{2} L_{\mathrm{o}}\right) /\left(2 K_{\mathrm{n}}\right)$

High impact velocities produce different fragments than a slowly applied collision of the same overall energy. The ratio of the impact velocity to the internal sound speed $\left(V_{0} / C\right)$ represents the ratio of the rate at which the energy of the impact is applied at the contact point to the rate at which it is carried away by elastic waves. Values of $V_{0} / C$ approaching unity imply that the impact energy is concentrated within a narrow elastic wave. Small values of $V_{0} / C$ correspond to nearly quasi static loading. The resulting parameter space is governed by the three dimensionless parameters: $E_{\mathrm{kln}} / E_{\mathrm{cr}}, V_{0} / C, v$.

Fig. 13 shows the simulation results of a single particle after impact on a plate. The results are compared with single particle breakage experiments (see Section 4.2).

\section{Results}

\subsection{Performance of grinding plant}

\subsubsection{Effect of high pressure air and solid feedrate}

In Fig. 14 the effect of the supplied pressure and solid feedrate on the $X_{50}$ of the mill product is presented, measured with the in-line cell for the open loop case. The hold-up dependence on these variables is shown in Fig. 15.

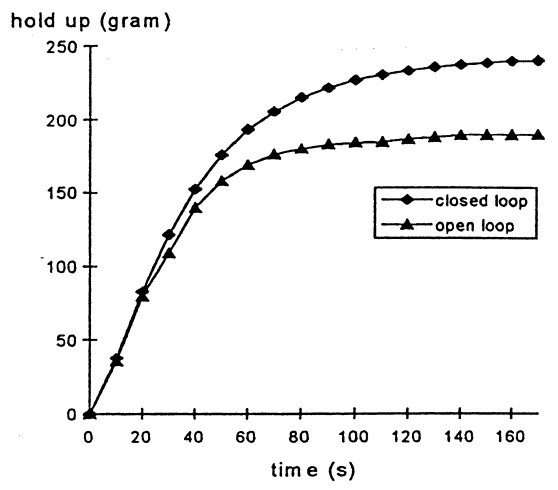

Fig. 16. Hold up depending on feedrate at constant nozzle pressure (simulation results). 


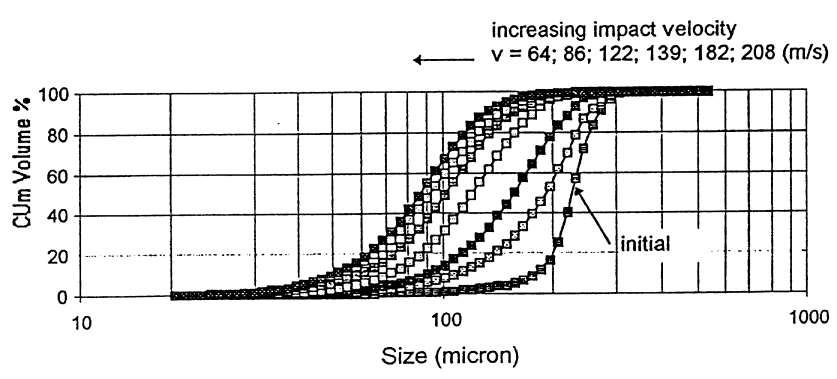

Fig. 17. Size distribution resulting from single particle impact. Material: Castor Wax, sieve size: $250-300 \mu \mathrm{m}$.

\subsubsection{Effect of the recirculating load}

When the classifier is recirculating a part of the mill product, this will influence the mill performance. The establishment of a new steady state is an iterative process [1]. In Fig. 16 the dynamic model shows the effect of the classifier on the $X_{50}$ and the hold up in comparison with the open loop configuration.

\subsection{Single particle breakage}

\subsubsection{Single particle breakage tests}

At "Université de Technologie de Compiègne" an instrument was used to study the impact breakage of single particles on a flat target [9]. An orifice immersed in a fluidized bed entrained particles in a gas flow which were accelerated in a nozzle. The particles impact on a target at a distance of $50 \mathrm{~mm}$ and at an angle of $90^{\circ}$.

In order to define the impact energy of the particle, the velocity of colliding particles was measured. A laser sheet is focused on the axis of the jet. The collision velocity was calculated from the reflected signal measured by emitterreceptor opticals that were located along the flow.

In Fig. 17 the cumulative distribution of the impacting particles is given for a Castor Wax sample impacted at 1,2 and $3 \mathrm{bar}$. This is to illustrate that there is a distribution in the impact velocity. The assumption made was that all impacting particles had the same velocity. Out of the impact tests the kinetic energy per unit of mass can be calculated with $v^{2} / 2$.

\section{Conclusions and discussion}

The influence of the feedrate and supplied pressure on the PSD of the end product and mill hold-up for the $10 \mathrm{in}$. mill are quantified. This experimental data is used for model verification.

The feedback control system on the feedrate or supplied pressure inputs is able to stabilize the $X_{50}$ of the end product at the desired level. For state estimation (e.g., hold-up) and modelbased control a more detailed model with a larger accuracy is required.

The hold up is an important internal variable influencing the grinding and particle transfer processes inside the mill. Model equations contain terms to take into account the hold-up effect.
The dynamic model produces realistic results for the stable operating range of the mill. When the mill is overloaded the calculated hold-up reaches values that are higher than the maximum possible value, causing the vortex to become unstable.

The classifier efficiency is determined by pilot plant experiment and fitted with a empirical curve. This efficiency curve is implemented in the dynamic model of the closed circuit grinding plant to predict the newly achieved operating conditions.

\section{Future research}

\subsection{Extension of the dynamic model}

The dynamic model will be extended from two to eight zones. The tangential and radial velocity components will be functions of place, hold-up (and therefore time) and supplied air. LDA measurements will be carried out to obtain data about the flow fields.

\subsection{Flow simulation of mill chamber}

Extensive simulation runs will be carried out to assess the dependence of the cell size, total number and initial PSD of particles and the time step on the simulation outcome. The model will be made three-dimensional instead of two-dimensional to investigate influences of the bottom and top walls on the internal classification and breakage behaviour of particles in the chamber. In the grinding zone the flow patterns of the separate jets will be superimposed on the vortex flow field.

\subsection{Measurement of flow patterns with laser doppler}

The measurement of root mean square turbulence and the probability of velocity is possible with laser Doppler technique. A perspex bottom plate was built to give access to the mill chamber.

\section{References}

[1] K. Heiskanen, Particle Classification, Chapman and Hall, London, 1993.

[2] R. Eek, Control and dynamic modelling of Industrial Suspension Crystallizers, Concept PhD Thesis, February 271995.

[3] E. Gommeren, Modelling of a closed loop jet mill, 8th European Symposium on Comminution, Stockholm, Sweden, May 1994.

[4] J.A. Talman, Computer simulations of a jet mill, Quarterly report for the Japanese-German Center Berlin, September 1994.

[5] A. vd Bos, The effect of material porperties on the grinding efficiency of a jet mill, Graduate Thesis, DuPont/TU Delft, December 1994.

[6] L.G. Austin, Introduction to the mathematical description of grinding as a rate process, Powder Technol. Rev. 5 (1971).

[7] R. Illner, Neunzert, H., On simulation methods for the Boltzmann equation, Transport Theory and Statistical Physics, 1987.

[8] Prof. C.S. Campbell, Department of Mechanical Engineering, University of Southern California, LA, USA, 1993.

[9] A.V. Potapov, C.S. Campbell, The two mechanisms of particle breakage and the velocity effect, Powder Technol. 93 (1997) 13-21. 